\title{
Whence Ontological Structural Realism?
}

\author{
Juha Saatsi* \\ School of Philosophy \\ University of Leeds \\ J.T.Saatsi@leeds.ac.uk
}

May 9, 2008

\begin{abstract}
'Structural realism' is a buzzword in the scientific realism debate. Various positions with diverse motivations fall under this label. A much advertised distinction is between epistemic and ontological forms of structuralism. This paper scrutinizes the alleged dichotomy between these two 'alternatives', and criticises the considerations that have been taken to motivate the ontic variety over the epistemic. I will argue that ontological structural realism is not called for within the traditional realism debate.
\end{abstract}

\section{Introduction}

'Structural realism' is a buzzword in the realism debate. Like bees buzzing after honeydew, thinkers are drawn to the flourishing variety of structuralism in the field of philosophy of science. The frantic activity has resulted in dozens of publications and, perhaps quite unavoidably, much ambiguity in what structural realism actually is, and what ultimately motivates it. The blossoming variety of different conceptions calls for some clarificatory work.

There's a much advertised distinction between epistemic and ontological varieties of structural realism. 'Ontic' Structural Realism (OSR) is motivated by considerations from the foundations of physics, and it is characterised as metaphysics. Epistemic Structural Realism (ESR), by contrast, represents a 'mere' epistemological refinement to 'standard' realism. This paper focuses on the motivations of OSR, and especially on the claim that in moving from standard realism to structural realism one should not stop before one arrives at the rather radical position of OSR. I claim that the advocates of OSR have failed to motivate it as an alternative to ESR and other 'non-standard' forms of realism. Although there's incentive to move away from object-oriented standard realism, there's no need to go as far as OSR.

${ }^{*}$ I wish to thank Steven French and Angelo Cei for helpful correspondence. 
Section 3 scrutinises the argument from the so-called metaphysical underdetermination. Section 4 looks at the way in which structuralism in philosophy of physics bears on the realism debate. Section 1 begins by making clear what ESR is, and what motivates it.

\section{What ESR is (not)}

There is a natural motivation for epistemic structural realism. It is the possibility of having 'the best of both worlds', as put by Worrall (1989), with regard to two opposing arguments at the core of the realism debate. The realist wants to paint an optimistic image of the truth content of our present theories that is plausible in the face of our best understanding of actual science and its method, motivated by the intuition behind the No Miracles argument, and not falsified by the history of science (Pessimistic Induction). This requires a principled way of delineating continuity in the truth content across radical theory shifts. ESR purports to identify the structural content of a theory in such a way as to ensure cumulative continuity in that kind of content.

The structuralist intuition springs from the fact that in various historical theoryshifts there are crucial mathematical equations that are carried over either intact or, more typically, as one set of equations being a limiting case of the other. There are examples in which the crucial equations of the successive theories are (a) formally identical, but furnished with divergent interpretations (e.g. the shift from Fresnel's ether theory to Maxwell's electromagnetic theory of optics); (b) formally equivalent apart from one or two new parameters, say, which disappear at some well-defined mathematical limit to yield the old equation (e.g. moving from the Galilean to the Lorentzian inhomogeneous group of transformations). The latter (more typical) cases get arguably further support from the endorsement of Heinz Post's (1971) general correspondence principle according to which any acceptable new theory should explain the well-confirmed part of its predecessor. Although this principle can be viewed to manifest itself in actual science in a variety of ways (Hartmann, 2002), an important dimension of Post's well-received notion of both descriptive and prescriptive correspondence in modern science is undeniably of the relevant mathematico-structural kind. Worrall's suggestion was to take the theoretical continuity manifested as such formal mathematical correspondence to be the locus of realist commitment.

This is a valid structuralist intuition. The highly mathematical nature of modern science together with the presumed descriptive soundness of the general correspondence principle makes it a very promising idea, at least with respect to some domains of science. But there remains much to be clarified to turn the intuition into a credible argument. To begin with the most obvious, the structuralist needs to ensure that the kind of continuity in focus really has to do with the realist rather than empiricist content. For surely the empiricist is also bound to find a level of continuity in the mathematical structures of a theory—namely those structures that 
encode the theory's empirical content. (Bueno, 1999; van Fraassen 2006) The realist claim is meant go further, of course, to declare a structural correspondence in the relevant theoretical content. Hence, Poincaré (as quoted in realist lights by Worrall, 1989) asserts that

The differential equations [in Fresnel's theory] are always true [that is, they are carried over into Maxwell's theory], they may always be integrated by the same methods and the results of this integration still preserve their value.

It cannot be said that this is reducing physical theories to practical recipes; these equations express relations, and if the equations remain true, it is because the relations preserve their reality. They teach us now, as they did then, that there is such and such a relation between this thing and that; only the something which we then called motion, we now call electric [displacement] current. But these are merely the names of the images we substituted for the real objects which Nature will hide for ever from our eyes. The true relations between these real objects are the only reality we can attain... (Poincaré, 1905: 161)

This is beautiful rhetoric, but what does this amount to in practise? To really see what 'the true relations' of Fresnel's theory are and how they compare with Maxwell's theory, one needs to engage in some history of science. (Saatsi, 2005) Worrall $(1989,1994)$ simply cites Fresnel's equations for the amplitudes of reflected and refracted polarized light, to point out that they are truly identical to those resulting from Maxwell's theory. But this is not enough. The motivation for going beyond empiricist commitments-the No Miracles argument-entails that we should be able to explain the success of the predecessor theory from the vantage point of the successor, in terms of truth-tracking theoretical content. This surely demands more than pointing out that the equations the two theories ultimately yieldthe equations that are used to test the theory against the experiment-are equivalent or stand in some limit-correspondence. What it demands, rather, is that we can account for the derivation of Fresnel's equation in terms of Maxwell's theory. For not only is there much to Fresnel's theorising besides 'the Fresnel equations' which represent the very end result of his theorising, but we also recall that the plausibility of the realist image, structural or otherwise, comes in part from fulfilling the intuition that success of a theory is connected to its approximate truth in a 'non-miraculous' fashion. This means that we should really be considering the relationship between the derivations by which the corresponding equations are arrived at in the first place, and it is not clear that this relationship is best understood in structural terms. (Cf. Saatsi 2005, 2008)

Another point to press the epistemic structural realist on concerns the sense in which one structure can be said to approximate another. Mere appeal to the general correspondence principle leaves this too open. The worry is that without a precise sense in which one structure corresponds to another we end up finding mathematical continuity where we want it. Even in the cases of intuitively appealing 
limit-correspondence we often have grave mathematical discontinuities that mark the theoretical revolution, as Redhead reminds us:

Consider the case of classical neo-Newtonian spacetime being replaced by the Minkowski spacetime of special relativity. We can consider a family of structures $\left\{S_{C}\right\}$ corresponding to varying the velocity of light $c$. For all finite $\mathrm{c}$ we can argue that the structure is stable with respect to changing $c$, but at $c=\infty$ there is a qualitative singularity in the sense that the metric of spacetime becomes singular in this limit. The existence of qualitative singularities of this type is also apparent in the case of the family of quantum mechanical structures indexed by a variable Plank's constant $\hbar$. (2001: 346)

Such discontinuities in the evolution of theoretical structures can perhaps be dismissed on the grounds that they are immaterial to the explanation of the success of the antecedent theory from the later perspective, but such claims need to be made on case-by-case basis and only after carefully scrutinising the nature of the particular structural (dis-)continuity in question.

In general it is quite clear that declarations of structural continuity cannot solely refer to mathematical equations, for we must (of course) somehow express the fact that the theories featuring these equations have the same subject matter. Formally equivalent equations are used for various purposes in different domains of science and any application of the correspondence principle needs to say something about how the equations in question are comparable apart from the shared logicosyntactic form. That is, we need to focus on interpreted equations. In some cases it is very straightforward to relate the equations to the same observable phenomenon (e.g. the Fresnel-Maxwell case), but we should ask whether there is a principled and preferred way of making the comparison. Is there a principled (logical) way of teasing out the structural content? In particular, do theories have to be regimented in some way for the comparison to be justified? For example, within the syntacticaxiomatic framework the prima facie possibility of using Ramsey sentences arises: these leave only mathematico-logical structure to supplement the content expressible with the terms left outside of the Ramsey-elimination. But arguably there are various reasons for preferring the alternative semantic framework of theories, and it turns out that this framework lends itself to a very different structuralist reading.

All in all, the thesis of ESR needs sharpening. Nevertheless, the epistemological motivation for structural realism is valid. The project of first making the structuralist proposal more precise and then comparing it to various instances of historical theory change is well-defined and intuitively cogent one. But it has been recently misinterpreted by some of the advocates of the alternative OSR. The key issue hinges on the proper interpretation of the epistemic humility of ESR. Let's take a typical statement of Worrall's realist position:

According to Worrall's [Epistemic Structural Realism] all that we know of the world is its structure, as exemplified in our scientific theories, 
and the 'nature' of the underlying elements (physical objects) remains 'hidden' in some sense. (Cei and French, 2006: 634)

The present question concerns the sense in which the nature of reality remains hidden in ESR. Cei and French (2006) offer an interpretation of Worrall's 'hidden natures'. They propose that we might equate this epistemic humility with the kind of Ramseyan Humility that David Lewis (forthcoming) has advocated, and they find the consequences of this equation problematic for the epistemic structuralist.

The suggestion, then, is that a cost of adopting the Lewisian picture would be increased pressure to locate the distinction between structure and nature with the latter and hence the necessity of facing up to French and Ladyman's arguments. (ibid., 652)

There is no need to explore in detail the kind of epistemic humility that arises in Lewis's metaphysics by virtue of combining a combinatorial principle of modality with primitive identity of properties ('quiddities'). It just needs to be pointed out that for Lewis the epistemological predicament of Ramseyan humility arises from the possibility that our final theories are in a sense multiply realisable. Cei and French explicitly acknowledge this, and it raises the question why they take this sort of epistemic humility to be a potential way of capturing Worrall's 'hidden natures'. For it is evident that Worrall (1989) is purely concerned with the antirealist argument from the history of radical theory-shifts, and not at all with the (rather idealised) notion of 'final theory'. Indeed, according to Worrall the ether theory of Fresnel's, for example, would describe the nature of light (were it true). So surely the final theory would not leave any nature (in Worrall's sense) hidden, if we were justified in taking the theory to be true! So why do Cei and French read Worrall this way? Perhaps it is partly due to Worrall's appeal to Poincaré, and Poincaré's appeal to Kant. ${ }^{1}$ But it needs pointing out that Worrall merely uses Poincaré's writings as a heuristic spring board to ESR, wholly disregarding his neo-Kantianism. Another source of motivation for Cei and French may be the fact both Lewis and Worrall (2001) appeal to Ramsey sentences as a way to capture structure-as-opposed-to-nature. But, horses for courses: Ramsey sentence is simply a piece of logical machinery that can be used for many philosophical purposes, and Lewis's purposes are far removed from Worrall's.

The motivation that Cei and French try to gather for OSR from their reading of 'hidden natures' fails. It is much more natural to attribute to ESR the following sense of epistemic humility. Our successful theories have often radically changed, so we are not in a position to commit to the full truth of our present theories. Rather, we should commit to our present theories being partially true in some 'structural' sense. With a suitable nature-structure distinction at hand we can say that our present theories, whether final or not, describe the structure of the world correctly,

${ }^{1}$ Lewis's Ramseyan humility is inspired by Rae Langton's (1998) interpretation of Kant. 
but not its nature. Our theories describe various properties of the worldly furniture and processes, and these properties describe a possible way the nature of these things could be. But we do not know that our world is a world of that kind. What we do know, however, is that the structure of our world-whatever its nature is - is such that it is correctly described by our theories. This sense of epistemic humility straightforwardly relates to the problem that allegedly 'inductively' rises from the history of science: most our current theories are probably not final onessomething that is also supported by the grand difficulties in making our theories fit together-and even if one of our theories is a final theory (for its domain) in some sense, we are simply not in a position to claim that we know that.

I take ESR to be a well-motivated, somewhat programmatic realist alternative. The advocates of OSR take this as their starting point, and then offer two distinctive sources of motivation for going beyond ESR. One turns on a particular kind of underdetermination arguably exhibited by some of our best theories, seriously impeding any substantial proclamation of realist commitments. The other source of inspiration comes from witnessing certain structuralist themes in the philosophy of physics, and develops into an argument by adopting a particular perspective on the relationship between metaphysics and epistemology. These two motivations for OSR are scrutinised in the next two sections.

\section{Metaphysical Underdetermination}

James Ladyman asked about structural realism: 'is it metaphysics or epistemology?' (1998: 410) As explicated above the answer seems clear: it is epistemology. There is, however, an interesting argument that at first seems to lead to a different conclusion.

\subsection{The argument}

Consider the challenge of providing a realist interpretation of quantum mechanics. Setting aside the problems with the collapse of the wave function to begin with, the realist should say of this most successful mature theory that it is probably approximately true in its claims about the unobservable world. So quantum particles, for example, are approximately like the theory tells us they are. But what does the theory tell us, exactly? Statistical behaviour of particles has been taken to be the key to their metaphysical nature. The behaviour of an assemblange of quantum particles is correctly described by either Bose-Einstein or Fermi-Dirac statistics, whilst Maxwell-Boltzmann statistics describes the behaviour of classical particles. What accounts for these differences in statistics?

According to our best understanding of quantum theory these particles can just as well be individuals ('cheese') or non-individuals ('chalk'), this metaphysical nature of the quantum objects being underdetermined by the theory. Both interpretations of the physics are equally compatible with the phenomena as well as the 
formalism. (French 1989, 1998; Huggett 1997; French and Rickles 2003; French $\&$ Krause 2006) So the realist is arguably in a pickle: she wants to say that the nature of quantum particles is as the theory says it is, but the theory doesn't say what it is!

We need to recognise the failure of our best theories to determine even the most fundamental ontological characteristic of the purported entities they feature. It is an ersatz form of realism that recommends belief in the existence of entities that have such ambiguous metaphysical status. What is required is a shift to a different ontological basis altogether, one for which questions of individuality simply do not arise. (Ladyman, 1998: 419-420)

I will now try to unpack this argument, assuming that there indeed is such metaphysical underdetermination at least with respect to some entities featured in our best physical theories. ${ }^{2}$ We can then focus on the question of how the realist should react. Also, I follow Ladyman \& French in taking standard realism to have the following metaphysical dimension: the ability to spell out our realist commitments in terms of objects, or entities, that exist. ${ }^{3}$ I do not take such metaphysical dimension to be a well-motivated part of realism. What I aim to show after explicating the argument from metaphysical underdetermination is that the move from standard realism to ontic structural realism is unnecessarily radical and not supported by the premise of metaphysical underdetermination. That is, it is natural to respond to the challenge by reducing the metaphysical dimension of standard realism, instead of adopting a radically alternative structuralist ontology.

But first, let's clarify the challenge itself: what is metaphysical underdetermination? For one thing, it is clearly different from the standard underdetermination objection to realism, according to which the realist cannot justify her commitment to any theoretical proposition $P$ since there is always an empirically equivalent incompatible theory which says $P^{\dagger}$ (incompatible with $P$ ). It is the rampant nature of this kind of underdetermination that (allegedly) makes it such a serious objection. If underdetermination was more limited in scope, so that only some theoretical propositions had empirically equivalent competitors, then realism about those parts of theories that are not thus underdetermined would be an option, at least prima facie. (Psillos, 1999: 167) Metaphysical underdetermination is different from empirical underdetermination by virtue of not being rampant. Rather, the former has a very limited scope: it is only the metaphysical nature of quantum particles (and whatever else leads to a similar predicament) that is underdetermined. So, prima

\footnotetext{
${ }^{2}$ Ladyman (1998), French \& Ladyman (2003) and French \& Rickles (2003) defend this premise particularly for quantum particles and quantum fields, and tentatively point towards the nature of spacetime. Pooley (2006) dissents, especially regarding the underdetermined status of spacetime points. See also Redhead \& Teller (1992) and Saunders (2003b) for criticism of the underdetermination thesis, and French \& Krause (2006) for further defence.

${ }^{3}$ Psillos (1999), for example, represents standard realism thus characterised for Ladyman \& French. French (2006) has called this 'object oriented' realism.
} 
facie, we should consider placing realist commitments to the common denominator, to whatever is common to both individuals-based and non-individuals-based interpretations of quantum physics, say.

But this strategy, the argument continues, is at a risk of leading to mere 'ersatz' realism. The worry is that in order to spell out one's realist commitments one needs to appeal to metaphysical natures. This follows directly from the metaphysical dimension of standard realism: to say that such-and-such entities exist requires that one spells out what an entity is. So, for example, if one says that 'According to QED there exist spin-half particles with charge $e^{\prime}$, one implicitly appeals to a metaphysical imagery (extrapolated from our experience of the macroworld) of point-like objects with properties mass, spin, etc., to give cognitive content to one's assertion. Assertions

(S) There are spin-half particles with charge $e$ and other properties as described by QED.

(S') There are hard elastic orange balls of the diameter of 24 centimeters, with a black stripe contouring around the ball.

are read on a par according to standard realism: they both assert the existence of some objects with some properties. Basketballs are observable, electrons are not, but we have good reasons to believe in the existence of both. So far so good. But the ontic structuralist points out that our epistemic grasp of the very objecthood of electrons, according to the argument from metaphysical underdetermination, is on a shaky ground. Therefore, $\mathrm{S}$ expresses no cognitive content beyond the surface semantic analogy which only pays lip service to the curious symmetry properties of the mathematical representation of quantum particles.

Hence, there is an acute problem with theoretical posits the metaphysical nature of which is underdetermined by the physics. ${ }^{4}$ The challenge is that the content of prototypical realist assertions regarding our knowledge of quarks and electrons, say, is deflated unless the realist is able to specify 'the most fundamental metaphysical categories' exemplified by the referents of 'quark' and 'electron'. The standard realist, not willing to tackle these subtle issues posed by the foundations of physics, is merely offering a cheap simulacrum of knowledge of the quantum world, based on an extraneous metaphysical image given in terms of categories

\footnotetext{
${ }^{4}$ The emphasis on theoretical posits is crucial here, as becomes clear by considering the following criticism of the above argument. Chakravartty (2003) has questioned the ramifications of the metaphysical underdetermination of individuality versus non-individuality by comparing it to the kind of underdetermination we face at the level of everyday objects: are tables and chairs ultimately just bundles of properties, or are they substances instantiating universals? French \& Ladyman (2003, p. 51) insist that the two cases differ since 'in the case of unobservables the content of belief in them is exhausted by their theoretical description - if that underdetermines their metaphysical nature then our belief is empty'. In my view there is a difference between the two cases, but I would also insist that agnosticism about the metaphysical natures of electrons, say, does not render realist commitments empty. There is much common ground to the alternative metaphysical pictures, which can be captured in terms of properties attributed to electrons.
} 
derived from our experience of the macroworld. Such realism is 'ersatz' in that it does not succeed in capturing any actual realist commitment regarding our best theories. What allegedly could save the realist, however, is ontological commitment to structure (as opposed to (non-)individual objects with properties) as the fundamental metaphysical category.

\subsection{Resisting the argument}

There are several points to be made in response to the above argument. (A) The common denominator-strategy of restricting the realist commitments to the shared parts of the underdetermined interpretations works. What is required is a move away from entities to properties in spelling out theoretical commitments. Labelling standard realism 'ersatz' may be appropriate in exposing the superfluousness of certain level of its implicit metaphysical commitments. But moving directly from standard (or 'object oriented') realism to structural realism is non sequitur. (B) To make sense of the impact of quantum mechanics in realist terms we do not need to defend realism about the metaphysical nature of quantum particles, say. (C) There is something fishy about the idea of avoiding metaphysical underdetermination by promising to provide yet another metaphysical framework of structures primitively understood.

A. If the standard realist is unable to choose between the metaphysically underdetermined options, are her realist commitments really as empty as the charge of ersatzism suggests? Is ontological structuralism a natural solution to her alleged predicament?

French and Ladyman press the standard realist on the nature of quantum particles:

[T] he (standard) realist is unable to give a full answer to [the question:]'what is a quantum object?', where a 'full' answer will involve the metaphysical nature explicated in terms of such fundamental categories as individuality, identity, etc. Van Fraassen rightly sees this as a challenge to standard realism (and it is regrettable that the standard realist has not seen fit to respond) expressing his conclusion as a waving 'good-bye to metaphysics' ${ }^{X}$ (1991, 480-482), leaving the field clear for constructive empiricism. (2003: 36, my italics)

So realism without adequate metaphysics succumbs to anti-realism. But perhaps to demand a full answer is to demand too much. Van Fraassen (as I read him) sees the kind of metaphysical underdetermination at issue to set a challenge for full-blown metaphysics, not realism per se. Underdetermination considerations in general do motivate van Fraassen's empiricism, for sure, but various degrees of epistemic confidence about the results of our inductive practices can be had whilst 
sharing some of van Fraassen's distaste for wholesale metaphysics. ${ }^{5}$

Nevertheless, French \& Ladyman insist (in a footnote marked by $X$ in the above quote) that 'if the realist refuses to be drawn on the metaphysics at least at the level of individuality versus non-individuality then how are we supposed to make sense of the impact of quantum mechanics?' (ibid., 50) I will look at the impact of quantum statistics below, but let's first consider this challenge in the abstract. There is an ambiguity here: there are two separate explanatory endeavours at stake for the realist. How can she explain (E1) the success of the theory by its partial truth; and (E2) what the world could be like to make the theory true simpliciter? The latter challenge asks what the world could be like according to our theory read literally, whilst the former asks what the world must be like according to our theory in order for the success of science (and of that theory in particular) not to appear 'miraculous'. Neither of these challenges is made insuperable by the metaphysical underdetermination at hand.

Regarding (E2), the realist can simply take different metaphysical frameworks to paint different meaningful images of how the world could be. Whether we have (ever could have) grounds to choose between such images- the very possibility and limits of metaphysical knowledge-is a different question, of course. ${ }^{6}$ Regarding (E1), the realist's response depends on her general characterisation of her realist commitments. What does it take to philosophically explain the success of a scientific theory in the spirit of scientific realism? How is the truth-tracking theoretical content that is doing the explaining to be delineated in the first place? Such questions surface in the context of the debate around pessimistic induction, and the realist — by virtue of not being ultra-optimistic about our current sciencetries avoid the force of the pessimistic historical record by appealing to some kind partial truth. I have urged elsewhere that this notion should be analysed in terms of theoretical properties responsible for successful derivations in science. (Saatsi, 2005) This conception of realist commitments is appropriate in the present context, too, since knowledge of these success-fuelling properties can be independent of having knowledge (or not) of the nature of reality in terms of the fundamental metaphysical categories relevant to the explanandum (E2) above. (I will illustrate this below with quantum statistics.)

It is unmotivated to label standard realism ersatz, with the implication that it amounts to no more than 'empty strutting and posturing' (as van Fraassen put it) unless salvaged by a novel metaphysical basis. In the face of recurrent theory-shifts

\footnotetext{
${ }^{5}$ Exactly how realism and metaphysics are related is a difficult question, of course. It is undeniable that some metaphysical assumptions play a role in scientific heuristics and decision-making, and perhaps some are required to deal with a different kind of underdetermination (Jones, 1991 vs. McMullin, 1992). I don't want to belittle these issues, but it is justified to bracket them here in order to focus purely on the argument from metaphysical underdetermination.

${ }^{6}$ Some argue that the realist is committed to optimism about the possibility metaphysics by virtue of strongly appealing to the inference to the best explanation, for example. (Hawley, 2006) I find these arguments problematic, but this doesn't matter here. What matters is that there is a further issue as to what extent realism and metaphysics are connected. And further issues need further arguments.
} 
the realist has qualified her commitments to the partial truth of our current theories. She also resolutely holds onto her basic argument for realism, the no miracles arguments. In the explanatory spirit of this argument the realist claims that theories' partial truth, suitably construed, is the best explanation for their success. I think we should construe a theory's partial truth as a matter of getting the (derivationally) crucial theoretical properties right. This construal is fit for the explanatory task, as far as certain historical case-studies are concerned. (Saatsi, 2005) This propertycentered (as opposed to object-centered) notion of partial truth can function similarly here. We can answer the question (E1) without taking a stance regarding the metaphysically underdetermined alternatives because the relevant explanatory, success-fuelling properties are shared by the competing metaphysical interpretations. We need to reject the intuition that the realist must engage in metaphysics to the extent that she can spell out her commitments in terms of fundamental metaphysical categories. So let's wave good-bye to standard realism, and welcome a metaphysically-less-inflated-realism, which I just call 'realism' from now on.

B. What, then, can a realist claim to know of quantum particles? How can these realist commitments be spelled out without reference to fundamental metaphysical categories? Consider, to begin with, a feasible metaphysical underdetermination vis-à-vis the nature of spacetime. The realist wants to explain the success of the general theory of relativity by claiming it to have correctly identified the curvature of spacetime as the source of gravitational phenomena. Explaining the successful accommodation of the precession of the Mercury perihelion in these terms is independent of the metaphysical question of whether the spacetime points of the substantivalist interpretation of GTR are to be understood haecceitistically or antihaecceitistically. ${ }^{7}$ In both of these metaphysical pictures the theory is true about the crucial unobservable features of the world, so that the concepts of curvature and geodesic, for example, similarly apply to properties of substantival spacetime. This kind of metaphysical underdetermination is quite different from the more oldfashioned empirical underdetermination that can take place in the spacetime context: one theory having a curved spacetime and the other having extra forces in its ontology. In the face of such underdetermination we really may not know what to believe in. Not so in the case of metaphysical underdetermination: we just believe that the theory correctly describes how spacetime is curved.

This case illustrates how metaphysical underdetermination can fall outside of natural realist commitments. But there is a crucial disanalogy to the case of quantum statistics: the underdetermined alternatives in the spacetime case-haecceitism and anti-haecceitism with respect to spacetime points-concern the modal identity of spacetime points, not their individuality. (Pooley, 2006) Nevertheless, the lesson

\footnotetext{
${ }^{7}$ The realist explanation of this success actually is independent of the existence or otherwise of spacetime points altogether, as corresponding to points of the mathematical manifold of a GTR model. The realist can remain agnostic of the 'fine-structure' of spacetime at the Planck-scale, only maintaining that the coarse-grained macrofeatures that emerge from the ultimate quantum theory of gravity are correctly described by GTR.
} 
generalises. We can account for the the success of quantum statistics without reference to the metaphysical nature of particles. The explanation is subtle, and I refer to Saunders (2006) for details. The gist of the explanation turns on the probability measure on quantum state space: the discreteness of this measure makes a crucial difference in how the states are counted under permutation symmetry. Saunders demonstrates how the difference between classical and quantum statistics arises from the fact that the probability measure is continuous for classical state space, whilst being discrete for quantum state space, even if both classical and quantum particles are assumed to be indistinguishable and permutation symmetry is applicable to both. (Permuting indistinguishable particles under permutation symmetry is taken to yield the very same state.) The realist does not have to deny that there may be different metaphysical explanations, underdetermined by the physics, for this crucial difference between classical and quantum systems. But these metaphysical musings go beyond what is required by the realist to explain 'the impact of quantum mechanics', as far as the explananda (E1) is concerned. Regarding this aspect of quantum mechanics, the realist is committed to the delineative discreteness of the quantum world - a property of quantum systems. Although this undeniably only scratches the surface of what the realist needs to say about quantum mechanics, it does address the source of the argument from metaphysical underdetermination.

C. Let's return to the two explananda (E1) and (E2), above. It seems that even at the level of (E2) the underdetermination does not motivate the radical step to OSR, regarded as 'offering a reconceptualisation of ontology, at the most basic metaphysical level, which effects a shift from objects to structures' (ibid., 37). Such a metaphysical project is in itself fully legitimate, of course, but cannot in my view gain any extra impetus from the metaphysical underdetermination. An ontological structuralist conclusion (regarding (E2)) could perhaps be be argued for by saying that structuralist metaphysics provides the only way to make sense of the notion of objecthood at the level of quantum particles. (Saunders 2003a, 2003b) ${ }^{8}$ But this is not the claim presently evaluated! Indeed, such a claim directly contradicts the underdetermination premise which is conditional on both horns being intelligible bona fide possibilities. If anything, it seems that the structuralist proposal only makes matters worse, for with such an alternative structuralist ontology available there would be three instead of two to choose from! ${ }^{9}$ The choice between these would presumably be done on the grounds of general metaphysical preferences. This, indeed, is another difference between metaphysical and empirical underdetermination; if one (pace van Fraassen) is optimistic about metaphysical reasoning in general, then arguably metaphysical underdetermination can be broken by con-

\footnotetext{
${ }^{8}$ Ladyman and Ross (2007) perhaps also argue for this claim, having shifted away from the argument from metaphysical underdetermination.

${ }^{9}$ It has been suggested that the individuals and non-individuals packages could be viewed as different representations of the common 'structuralist core' but this intuition must be substantiated in order to show how the underdetermined options go over and above the common core, instead of just being metaphysical alternatives.
} 
siderations that go beyond physics and belong to philosophy simpliciter.

$$
\text { *** }
$$

I conclude that the motivation gained from the metaphysical underdetermination for structural realism, and for ontological structural realism in particular, is highly problematic. I will next briefly look at an oblique line of enquiry that is sometimes taken to provide further grounds for OSR, or even for taking OSR to supplant ESR.

\section{Structuralism in Philosophy of Physics}

I now want to argue in more general terms for a distinction to be made between two levels of structuralist philosophy often run together in a synergistic fashion. ${ }^{10}$

One family of structuralist thought belongs to the philosophy of physics proper: the unifying theme is the conviction that the ontology of physics is best conceived in structural terms. This line of thought is well represented in the history of philosophy by the likes of Cassirer and Eddington, for example, as a way of philosophically refining the worldviews imposed upon us by quantum mechanics and the general theory of relativity. (French 2003, French \& Rickles 2006, Ryckman 2004) Very broadly speaking this movement can be characterised as an attempt to shift one's ontology away from objects, as traditionally conceived, and towards structures relationally understood. The historical as well as the contemporary literature on this line of thought is by and large spurred on by the central role of fundamental symmetries exhibited by our best physical theories: the diffeomorphism invariance of the general theory of relativity, permutation symmetries in quantum mechanics, gauge symmetries of gauge field theories, and so on. Very crudely put, these symmetries can in a sense be understood to 'relationally define', or 'constitute' the objects that are invariant under these symmetries, and hence the symmetries are ontologically prior to the objects in some sense.

A different set of structuralist ideas belongs to epistemology, and concern the question of what we can claim to know of the (mind independent) world. Again, there are eminent historical figures to draw on, such as Russell (1927) fighting against phenomenalism about the external world. But in the contemporary context the epistemological motivation, as outlined in section 2, boils down to something quite specific. It is the attempt to craft a plausible image of science, motivated by the intuition behind the No-Miracles Argument and not refuted by the history of science. Once again, the idea simply is, crudely put, that theories by and large get the 'structure' right but may well say falsehoods about the rest.

\footnotetext{
${ }^{10}$ It is not always easy to prise apart the different motivations running in parallel, but in my view an illegitimately close connection between different structuralist motivations is implied in Ladyman (1998), French \& Ladyman (2003), Saunders (2003b), Lyre (2004), and French \& Rickles (2006), for example.
} 
On the face of it, it is not easy to say exactly how structuralism in the philosophy of physics should interact with this epistemological idea. One might at first think that if the preferred ontology of physics is structural-so that one is an ontological structural realist at the level of philosophy of physics-then one must also be a structuralist with respect to one's epistemological scientific image, since all theoretical truths are ultimately truths about structure. But the connection isn't this straightforward. After all, the structuralist ontology is inspired by metaphysical questions regarding a literal reading of our best theories-questions such as: what are the spacetime points quantified over in GTR like; how to understand the nature of quantum particles in the face of the permutation symmetry, or the gauge symmetry behind the Bohm-Aharonov effect. The epistemological humility of the realist image, on the other hand, is based on the belief that our theories may only be partially true. Therefore the notion of partial truth adopted by the realist can affect whether or not a literal reading of our present theories has input on the realist's epistemic commitments. For example, it might be part of the realist image that there really is a curved spacetime and that free particles move along the shortest paths as mathematically represented by geodesics on a manifold-i.e. the theoretical terms 'curvature of spacetime' and 'shortest path' do refer-irrespective of whether the most fundamental spacetime ontology consists of dimensionless points or of something else completely. GTR might be a true representation of the curvature properties of spacetime whilst being a false representation of its 'fine structure'. Indeed, being a classical (non-quantised) theory this is most probably the case, as acknowledged by an epistemically cautious realist. Whether or not there is an argument for interpreting GTR substantivalism in structuralist terms, it is not clear what ramifications this argument should have on such a realist.

This example is enough to sever intimate link between ontological and epistemological structuralism. Structuralism in metaphysics might be appropriate for an interpretation of some theory $T$, but if the realist is only committed to $T$ being partially true it is not clear what epistemological lessons we should draw from the metaphysics. The realist only needs the resources required to capture those aspects of the world that were latched onto by the scientific practice in producing the successes of $T_{S}$. I believe that those features can be described independently of the underlying 'fundamental metaphysical categories'. Strangely enough, Ladyman and French also repeatedly stress that the preferred ontology cannot be drawn directly from the physics: 'we cannot infer the appropriate metaphysics for describing the world from the physics itself.' (da Costa \& French 2003: 188) ${ }^{11}$ So what becomes of the idea that the realist needs to be a structural realist in order to latch onto the world as described in physics?

I have been critical of the idea that taking into account the metaphysical conundrums of modern physics leads to structural scientific realism. There is another,

\footnotetext{
${ }^{11}$ They underscore this in connection of the metaphysical underdetermination, of course, to motivate the alternative structuralist ontology. As far as I can see, the alluded to ontological notion of structure forms just another fundamental metaphysical category.
} 
stronger idea that also ought to be dismissed. This line of thought urges that a structuralist ontology at the level of physics does not only motivate structural realism, but surpasses the arguments for ESR altogether. Lyre (2004), for example, writes in connection of gauge symmetries:

[A] philosophical view such as structural realism gains by far more credence if supported by arguments from science directly than by mere indirect and notoriously debatable considerations of the philosophy of science. This is the difference in style between the Worrall-type of arguments in favor of structural realism and the French-type of arguments - on the basis of the ontology of quantum theory-or the Stachel-type —on the basis of general relativity. (Lyre 2004: 621)

But this seems a grand non sequitur: epistemic structuralist à la Worrall (1989), for example, would not consider one or another interpretation of the literal reading of quantum mechanics to be of significance to his realism, since he is only committed to the claim that (a) the relevant theoretical structure suitably approximates the structure of any future theory of quantum phenomena, and (b) we can ultimately make sense of the success of quantum theory in terms of ESR.

The failure to properly distinguish between the ontological and epistemological levels of structuralist endeavour has landed the realism discussion in this context in a muddle. Lyre, for example, takes structural realism to be a monolithic position supported by arguments from both philosophy of physics and philosophy of science. These two sets of arguments simply do not have the same objective. One can quite consistently advocate a structuralist ontology vis-à-vis some feature of physics, and maintain ESR or some other realist position with respect to one's epistemological stance.

\section{Conclusion}

Several considerations for various forms of structural realism have been recently advanced in the quickly burgeoning literature. There is growing need to draw critical distinctions in order to regiment the multifaceted debate: too often different senses of 'structure' and 'structuralism' are confusingly placed under one and the same heading. Here I have attempted to make some headway with this clarificatory task, by focusing on different motivations for adopting a form of structural realism. If correctly interpreted, the original epistemic strand of structural realism is a well motivated, if still somewhat programmatic position. What has been hailed by some as the radical alternative - the ontic version of structural realims-is rather weakly motivated in comparison. Whilst there is most certainly room for various forms of structuralism in metaphysics and philosophy of physics, the links between the various considerations are most subtle than is currently aknowledged in the literature. 


\section{References}

Bueno, O. (1999). What Is Structural Empiricism? Scientific Change in an Empiricist Setting. Erkenntnis, 50(1):59-85.

Chakravartty, A. (2003). The Structuralist Conception of Objects. Philosophy of Science, 70(5):867-878. PSA Proceedings.

Dorato, M. and Pauri, M. (2006). Holism and Structuralism in Classical and Quantum GR. In Structural Foundations of Quantum Gravity. Oxford University Press, Oxford.

French, S. (1989). Identity and Individuality in Classical and Quantum Physics. Australasian Journal of Philosophy, 67:432-446.

French, S. (1998). Identity and Individuality in Quantum Theory. Stanford Encyclopedia of Philosophy.

French, S. (2006). Structure as a weapon of the realist. Proceedings of the Aristotelian Society, 106:1-19.

French, S. and Krause, D. (2006). Identity and Individuality in Modern Physics. Oxford University Press, Oxford.

French, S. and Ladyman, J. (2003). Remodelling Structural Realism: Quantum Physics and the Metaphysics of Structure. Synthese, 136(1):31-56.

French, S. and Rickles, D. (2003). Understanding Permutation Symmetry. In Brading, K. and Castellani, E., editors, Symmetries in Physics: Philosophical Reflections, pages 212-238. Cambridge University Press, Cambridge.

French, S. and Rickles, D. (2006). Quantum Gravity Meets Structuralism: Interweaving Relations in the Foundations of Physics. In Structural Foundations of Quantum Gravity. Oxford University Press, Oxford.

Hartmann, S. (2002). Essay Review: On Correspondence. Studies in History and Philosophy of Modern Physics, 33:79-94.

Huggett, N. (1997). Identity, Quantum Mechanics and Common Sense. Monist, 80:118-130.

Jones, R. (1991). Realism about what? Philosophy of Science, 58:185-202.

Ladyman, J. (1998). What is Structural Realism? Studies in History and Philosophy of Science, 29A(3):409-424.

Ladyman, J. and Ross, D. (2007). Every Thing Must Go: Metaphysics Naturalized. Oxford University Press, Oxford. 
Langton, R. (1998). Kantian Humility. Oxford University Press, Oxford.

Lewis, D. (Forthcoming). Ramseyan humility. In Braddon-Mitchell, D. and Nola, R., editors, The Canberra Plan.

Lyre, H. (2004). Holism and structuralismin U(1) gauge theory. Studies in History and Philosophy of Modern Physics, 35:597?624.

Musgrave, A. (1992). Discussion: Realism About What? Philosophy of Science, 59:691-697.

Poincaré, H. (1905). Science and Hypothesis. Dover, New York, reprinted 1952 edition.

Pooley, O. (2006). Points, Particles, and Structural Realism. In French, S., Rickles, D., and Saatsi, J., editors, The Structural Foundations of Quantum Gravity. Oxford University Press, Oxford.

Post, H. (1971). Correspondence,Invariance and Heuristics. Studies in History and Philosophy of Science, 2:213-255. Reprinted in S. French and H. Kamminga (1993).

Psillos, S. (1999). Scientific Realism: How science tracks the truth, chapter 4, pages 70-97. Routledge, London.

Redhead, M. (2001). The Quest of a Realist. Metascience, 10:341-347.

Redhead, M. and Teller, P. (1992). Particle labels and the theory of indistinguishable particles in quantum mechanics. British Journal for the Philosophy of Science, 43:201-218.

Ryckman, T. (2004). The Reign of Relativity: Philosophy in Physics 1915-1925. Oxford University Press, Oxford.

Saatsi, J. (2008). Eclectic realism - the proof of the pudding: a reply to Busch. Studies in History and Philosophy of Science. Forthcoming.

Saatsi, J. T. (2005). Reconsidering the Fresnel-Maxwell case study. Studies in History and Philosophy of Science, 36:509-538.

Saunders, S. (2003a). Physics and Leibniz's Principles. In Brading, K. and Castellani, E., editors, Symmetries in Physics: Philosophical Reflections, pages 289308. Cambridge University Press, Cambridge.

Saunders, S. (2003b). Structural Realism, Again. Synthese, 136(1):127-133.

Saunders, S. (2006). On the explanation for quantum statistics. Studies in the History and Philosophy of Modern Physics, 37:192-211. 
van Fraassen, B. C. (1991). Quantum Mechanics: An Empiricist View. Oxford University Press, Oxford.

van Fraassen, B. C. (2006). Structure: Its Shadow and Substance. British Journal for the Philosophy of Science, 57(2):275-307.

Worrall, J. (1989). Structural Realism: The Best Of Both Worlds? Dialectica, 43:99-124.

Worrall, J. (1994). How to Remain (Reasonably) Optimistic: Scientific Realism and the "Luminiferous Ether". In Hull, D. and Forbes, M., editors, PSA 1994, volume 1, pages 334-342. Philosophy of Science Association, East Lansing.

Worrall, J. and Zahar, E. (2001). Ramseyfication and Structural Realism. In Zahar, E., editor, Poincaré's Philosophy: From Conventionalism to Phenomenology, pages 236-251. Open Court Publishing Co., Chicago. 